\title{
Even experts cannot agree on the optimal use of platelet-rich plasma in lateral elbow tendinopathy: an international Delphi study
}

\author{
Jonathan P. Evans ${ }^{1,2^{*}} \mathbb{0}$, Nicola Maffulli ${ }^{3,4,5}$, Chris Smith ${ }^{1}$, Adam Watts ${ }^{6}$, Jose Valderas ${ }^{2}$ and Vicki Goodwin ${ }^{7}$
}

\begin{abstract}
Background: Platelet-rich plasma (PRP) is widely used in the management of lateral elbow tendinopathy (LET) despite conflicting evidence on its effectiveness. With high levels of user experience, this study aimed to assess consensus amongst experts on its clinical use.

Methods: A three-round international Delphi study was conducted. Participants were invited through national society mailing lists and contact lists derived from a systematic search of the literature on PRP. In round one, a primary working group developed 40 statements on PRP preparation and clinical application. In rounds two and three, an international group of researchers on PRP and clinical users of the device scored their levels of agreement with the statements on a five-point scale. Consensus was defined as an interquartile range of $\leq 1$.

Results: Consensus of agreement was only reached for $17 / 40$ (42.5\%) statements. For statements on PRP formulation, consensus of agreement was reached in $2 / 6$ statements (33\%). Only limited consensus on the contraindications, delivery strategy and delivery technique was achieved.

Conclusion: Experts reached very limited consensus on the use of PRP in LET. High levels of user experience have not resulted in a convergence of opinion on the technical components of PRP formulation and delivery, substantiating the need for further studies and improved trial reporting.
\end{abstract}

Keywords: Platelet-rich plasma, PRP, Consensus, Elbow, Tendinopathy, Tennis elbow

\section{Background}

The use of platelet-rich plasma (PRP) for the treatment of lateral elbow tendinopathy (LET) has rapidly increased over the last few years [1]. Tendinopathy is a multifaceted condition, with varied and highly complex inflammatory system modulation, and great variability in patients, age and location of presentation, and chronicity of the condition. Biological therapies are being promoted as a potential beneficial modality that seeks to mediate the dysfunctional biology of tendinopathy by boosting healing mechanisms [2]. Both animal and in vitro studies

\footnotetext{
*Correspondence: j.p.evans2@exeter.ac.uk

1 Royal Devon and Exeter NHS Foundation Trust, Barrack Road, Exeter, UK Full list of author information is available at the end of the article
}

suggest that the anabolic effect of growth factors released from the platelets in PRP promote tendon matrix repair [3-7]. However, clinical studies have not produced univocal results: the latest Cochrane review suggests no evidence to support the use of PRP for the management of musculoskeletal soft tissue injuries [8]. For LET, systematic reviews and meta-analyses have produced conflicting results on PRP efficacy $[1,9]$.

Much of the criticism directed towards PRP research relates to the heterogeneity of patient selection, PRP preparation, and administration techniques [1, 2, 8-10]. Furthermore, inconsistency of protocol reporting, outcome measurement, and adverse events reporting has hindered consistent interpretation of treatment effects or harm [11]. 
PRP is prepared through concentration of the patient's own blood. However, depending on the equipment, the protocol used and the patient's own blood profile, highly variable concentrations of platelets, erythrocytes, and leukocytes are obtained [12]. Administration variability relates to patient selection, the frequency and interval of administration, the use of adjunct ultrasound guidance, and post-injection protocols. Furthermore, the use of local anaesthetic, platelet pre-activation, anticoagulation and $\mathrm{pH}$ buffering are not standardised.

The lack of treatment standardisation may, in part, be related to the regulation of biological therapies of this nature. Unlike medicinal products, PRP, as an autologous therapy, has not been subject to the standardised methodology of phased trials. Though the preparation apparatus (e.g., centrifuge system) may have been subject to pre-market approval, the product to be administered to the patients (i.e., PRP) has not been standardised. Hence, the treatment constituents, dose, administration and adverse events have not been investigated according to the standards applied to medical drugs.

A large-scale trial of a methodology in accordance with a phase 2 clinical drug trial may be able to elucidate the optimal PRP constituents, dose and administration, but would require considerable resources and financial support. With the widespread use of PRP within both research and clinical communities, an alternative approach may be to explore expert opinions, whose personal techniques may have evolved and whose collective experience may have converged, in an attempt to gain consensus using a validated methodology.

The Delphi method uses a series of sequential question sets (or rounds), interspersed with controlled feedback to gain the most reliable consensus of opinion from a group of experts [13]. The Delphi method is particularly useful where individual judgments need to be assessed and combined to address a lack of agreement or incomplete knowledge [14-16]. In addition to identifying a treatment consensus (or lack thereof), the technique can also inform research priorities where knowledge deficits exist [15]. The Delphi method has been extensively used in healthcare settings, and has recently been employed in expert consensus exploration of Achilles tendinopathy [17] and shoulder rotator cuff pathology [18].

This study aimed to elicit opinion and assess levels of consensus on the use of platelet-rich plasma in lateral elbow tendinopathy. In the absence of phased trial data, exploration of expert consensus is justified. Patterns in treatment practice or, indeed, lack thereof may assist in the future formulation of study protocols and treatment guidelines.

\section{Methods}

\section{Study design}

This study used a three-stage Delphi technique. This methodology has been chosen to develop criteria that are based on consensus gained from an expert panel, where insufficient quality and grade of evidence exists to develop evidence-based criteria [19].

\section{Participant selection and recruitment}

A steering group was recruited to undertake round one through personal communication with the lead author (JE). This group of 10 individuals was composed of upperlimb orthopaedic surgeons, a musculoskeletal radiologist, and PRP researchers. All were invited directly. The steering group was utilised in round one and was asked to participate in the subsequent rounds.

The subsequent rounds recruited the expert opinion of a larger cohort of participants. To increase the heterogeneity of the expert panel, including the desire to gain national and international opinion, two approaches were used to invite both research-based and clinical-based individuals with experience in PRP application. The research cohort was recruited through a database search of published articles on PRP followed by email communication with the lead author; the clinical cohort was recruited through national society mailing lists.

Database searches were undertaken on 15 February 2017 in Medline, EMBASE and CINHAL databases from inception to the present, using modified British Medical Journal (BMJ) research filters for systematic reviews, randomised controlled trial, cohort and case-control studies (Additional file 1). Following title and abstract review, 57 articles were identified. Where possible, contact email addresses were obtained by contacting the corresponding author; lead authors were then invited to participate in the Delphi process via email.

To capture clinical users of PRP who may not be actively involved in research, the European Society for Surgery of the Shoulder and Elbow (SECEC), the British Association of Sports and Exercise Medicine (BASEM) and the British Society of Musculoskeletal Radiologists were invited to participate via a mailout of society contact lists.

\section{Expert panel size}

The question of participant numbers is dependent on the minimally sufficient number to constitute a representative pooling of judgments [20]. Wide variations in expert numbers have been reported in Delphi studies [15], though nominally they tend to be within 20-60 [21].

The study aimed to recruit a minimum of 25 experts. The primary group of experts in round one was 10 . In round two, a minimum of 20 additional experts was 
required for study progression. Round three required retention of $>60 \%$ of respondents. Though it has been reported that the reliability of composite judgments increases with respondent numbers, there is little empirical evidence on the effect of participant numbers on reliability or validity of the consensus process [16] if the panel composition is appropriate.

\section{Procedure}

\section{Round one}

The initial steering group was contacted through the Bristol Online Survey electronic portal (BOS, University of Bristol, Bristol, UK). This expert panel was presented with open questions based on the domains of PRP reporting identified by Murray et al. [22]. These open questions asked for the participants' opinions on the themes of patient selection, PRP preparation and delivery, postprocedural care and outcome assessment (Additional file 2). Consolidation of text answers was undertaken through content analysis. The answers were read by two assessors $(\mathrm{JE}+\mathrm{CS})$ and, from the response themes, a list of 40 statements was developed for agreement scoring in round two. In cases of disparity between participants, the predominant theme was utilised. The established statements were distributed amongst the study authors for the assessment of thematic structure and comprehension.

\section{Round two}

The statements from round one were developed into an electronic questionnaire, with each statement requiring an agreement score using a Likert scale from 1 (strongly disagree) to 5 (strongly agree). The questionnaire was distributed to all those who responded to the invitation sent to PRP researchers, national society members and those from round one willing to be involved in subsequent rounds. Basic demographic information on the participant was collected to quantify participant PRP experience (clinical user/PRP researcher/both), user occupation (researcher/surgeon/radiologist/sports physician), and total number of PRP injections for LET administered annually. Participants were also able to suggest edits to the statements using a free text option. Statements were modified if suggestions by more than three participants were thematically similar and not in contrast to the predominant group response.

\section{Round three}

Participants were sent an individualised feedback report including their round two scores and the group's scores for each statement, represented using a histogram. Participants were able to reflect on their scores and, if necessary, change their score whilst maintaining anonymity. The same Likert scale was used for round 3. The benefit of continuing the iterative process was assessed following round 3 completion using standardised stopping criteria.

\section{Ethical considerations}

This study was granted ethical approval by the University of Exeter Medical School review panel (Nov16/B/105). The iterative nature of the Delphi technique meant that the participants remained anonymous to each other, but not to the research team [21].

\section{Data analysis}

Quantitative analysis of Likert ratings was undertaken using Stata (StataCorp. 2015. Stata Statistical Software: Release 14. College Station, TX: StataCorp LP). For measures of central tendency and level of dispersion, median and interquartile range (IQR) are strongly favoured when Likert scales are used $[19,21]$. The consensus criterion for this study was an interquartile range (IQR) of one or less. An IQR of less than one means that more than $50 \%$ of all opinions fall within one point on the scale [23]. For those with an IQR of $\leq 1$, the median score was taken as the agreement level and statements grouped into one of three categories: consensus of agreement (median score 4 or 5), consensus of disagreement (median score 1 or 2) or consensus of 'neither agree nor disagree' (median score of 3). Percentage agreement (participants answering either four or five on the Likert scale) is also presented. The stopping criteria at round 3 was assessed using the parametric method of coefficient of variation difference (CVD) [24]. This was calculated by subtracting the individual item $\mathrm{CV}$ (standard deviation/mean) from round three, from the corresponding CV from round two. A value close to zero denotes stability of responses with a cut-off value of $>0.5$ deemed a decision limit for a need for further rounds [23]. Post hoc analysis of response differences between different groups of PRP user experience (clinical user or clinical researcher/pure researcher), clinical occupation (radiologist/physician or surgeon) and injection number per annum (low volume $\leq 20$ or high volume $>20$ ), was undertaken at the statement level using a non-parametric Mann-Whitney $U$ test. Statistical significance was set at $p<0.05$.

\section{Results}

\section{Participants}

The primary working group consisted of 10 participants, of whom nine were senior orthopaedic surgeons and one a senior radiologist. Half of the group were actively involved in PRP research as well as clinical practice. The multimodal recruitment strategy resulted in a further 28 participants, with a total of 38 participants from 14 different countries, completing the round two questionnaires. Following the return of round two feedback and 
two email reminders, round three was completed by 28 participants (74\% response rate). The group completing round three consisted of three radiologists, seven sports physicians, one researcher who did not undertake a clinical role and 17 orthopaedic surgeons. Of this group, 12 (43\%) were actively involved in PRP research. As a marker of research impact demonstrated by the research active participants, their h-index was extracted from the Scopus ${ }^{\circledR}$ (Elsevier B.V.) database yielding a median h-index of 13 (range 5-83). The annual use of PRP injection for LET was grouped into five categories: nine participants $(25 \%)$ administered $\leq 5$ injections per annum, two (7\%) administered 5-10, eight (26\%) administered 10-20, five (18\%) administered $20-50$ and six (21\%) administered $>50$ per annum.

\section{Consensus development}

Between rounds two and three, two statements were modified following free text feedback from multiple participants. Statement 2 was changed from "PRP should only be considered following 6-months of conservative therapy" to "PRP should only be considered following at least 3 months of conservative therapy", and statement 24 was changed from "A $19 \mathrm{~g}$ needle is the recommended minimum size used to administer PRP" to "A 19 g needle is the recommended MAXIMUM size used to administer PRP".

Stability between rounds two and three was assessed and confirmed using the coefficient of variation difference (mean CVD $-0.03, \mathrm{SD} \pm 0.04$, range -0.11 to 0.04 ). Further iterations of the questionnaire were therefore deemed unlikely to result in further change.

Consensus of agreement (IQR $\leq 1$, median score 4 or 5 ) occurred for $17 / 40$ statements (42.5\%) (Table 1). Consensus of disagreement (median score 1 or 2) occurred for $2 / 40$ statements $(5 \%)$. Consensus of 'neither agree nor disagree' (median score 3 ) occurred for $4 / 40$ statements $(10 \%)$. Consensus was not reached for $16 / 40$ statements (40\%) (Table 2).

\section{Group analysis}

Assessment of statistically different median scores between 'PRP user experience' groups revealed differences in opinion for statement 2 ("PRP should only be considered in patients who are experiencing considerable intrusion into their activities of daily life") with clinical users scoring a median of 3 and clinical researchers/pure researchers scoring a median of $4.5(p=0.007)$. For occupation type, grouped into two categories of radiologist/ physician or surgeon, statistically different median scores were found for statement 3 ("PRP should only be considered in patients who are experiencing considerable intrusion into their activities of daily life") [3 vs 4 respectively
( $p=0.04)$ ], statement 25 ("Ultrasound guidance should be utilised in all PRP injections for Lateral Elbow Tendinopathy") [5 vs $4(p=0.03)$ ], statement 26 ("Needle fenestration is recommended over a single injection technique") (4.5 vs $4[p=0.01)]$ and statement 30 ("Surgery is recommended for patients in whom PRP treatment is not effective") [3 vs $4(p=0.004)]$. No differences in statement scores were noted between low-volume users $(0-20$ per annum) and high-volume users ( 20 to $>50$ per annum).

\section{Discussion}

This study attempts to assess levels of expert consensus on the use of platelet-rich plasma in patients suffering from lateral elbow tendinopathy. The use of PRP is increasing despite inconclusive evidence of clinical efficacy [25]. This expansion in the use of PRP likely arose both from the availability and inherent safety of this technology, and the desire to offer a therapy for a condition which is recognised as challenging to treat, with limited evidence of a gold standard treatment approach [11]. In a situation of lack of data regarding clinical efficacy, but widespread use, it was deemed both legitimate and practical to assess expert consensus. This study has identified some commonality in patient selection, administration techniques and post-procedural follow-up care. However, it also demonstrates the wide variations in expert approach to PRP production and formulation, application of local anaesthesia, use of imaging adjuncts, and its interplay with non-steroidal anti-inflammatory drugs (NSAIDs) and corticosteroids.

Statements involving patient selection demonstrated adequate consensus, with agreement that participants can be selected if they have characteristic LET pain, are over the age of 18 years and are experiencing considerable negative impact of LET on their lives. Furthermore, there was agreement that occupation or level of demand on upper limb function should not be a factor in participant selection. The statement within this section which did not reach consensus related to the duration of symptoms, given the wide score dispersion apparent with an IQR of 2.5. Furthermore, this statement was altered between rounds because of feedback from multiple participants to lower the duration of conservative therapy from 6 to 3 months. Though this shifted the median from 3 (round two) to 4 (round three), significant dispersion remained. A significant majority of PRP studies have concentrated on chronic LET ( $>6$ months of symptoms), and this lack of consensus may highlight an area of future research on the effect of PRP at an earlier stage in the course of LET.

There is limited evidence available on both the potential contraindications to PRP delivery and the potential 
Table 1. Results of round 3

\begin{tabular}{|c|c|c|c|c|c|}
\hline Statement & $\underline{\mathrm{IQR}}$ & $50 \%$ & $100 \%$ & Median & \%Agreement \\
\hline $\begin{array}{l}\text { 1. PRP should only be considered in patients presenting with characteristic } \\
\text { tennis elbow pain }\end{array}$ & 1 & & & 4 & 89.29 \\
\hline $\begin{array}{l}\text { 2. PRP should only be considered following at least } 3 \text { months of conservative } \\
\text { therapy }\end{array}$ & 2.5 & & & 4 & 64.28 \\
\hline $\begin{array}{l}\text { 3. PRP should only be considered in patients who are experiencing } \\
\text { considerable intrusion into their activities of daily life. }\end{array}$ & 1 & & & 4 & 64.29 \\
\hline $\begin{array}{l}\text { 4. PRP treatment can be considered in patients over the age of } 18 \text {, with no } \\
\text { upper age limit. }\end{array}$ & 1 & & & 5 & 96.43 \\
\hline $\begin{array}{l}\text { 5. PRP treatment can be considered in patients with manual or sedentary } \\
\text { occupations. }\end{array}$ & 0.5 & & & 5 & 96.43 \\
\hline $\begin{array}{l}\text { 6. PRP treatment can be considered in both high demand (e.g. sports people) } \\
\text { and low demand (e.g. office worker) patients. }\end{array}$ & 0.5 & & & 5 & 100 \\
\hline 7. PRP is contraindicated in patients with a coagulopathy. & 1 & & & 2 & 21.43 \\
\hline 8. PRP is contraindicated in patients with large wrist extensor tendon tears. & 2 & & & 2 & 28.57 \\
\hline 9. PRP is contraindicated in patients taking anticoagulant medication. & 1 & & & 2 & 21.42 \\
\hline $\begin{array}{l}\text { 10. PRP is contraindicated in patients with a dependence on Non-Steroidal } \\
\text { Anti-Inflammatory Drugs (NSAIDs). }\end{array}$ & 3 & & & 3 & 42.85 \\
\hline $\begin{array}{l}\text { 11. PRP is contraindicated in patients with known thrombocytopenia (less } \\
\text { than } 150,000 \text { platelets per microlitre of whole blood). }\end{array}$ & 1 & & & 4 & 51.85 \\
\hline $\begin{array}{l}\text { 12. PRP is contraindicated in patients who have received a steroid injection for } \\
\text { LET, within } 3 \text { months of the intended PRP treatment date. }\end{array}$ & 2 & & & 3 & 42.85 \\
\hline $\begin{array}{l}\text { 13. The minimum recommended platelet concentration of injected PRP is } 2 x \\
\text { baseline. }\end{array}$ & 1.5 & & & 3.5 & 50 \\
\hline $\begin{array}{l}\text { 14. The maximum recommended platelet concentration of injected PRP is } 5 x \\
\text { baseline. }\end{array}$ & 1 & & & 3 & 39.29 \\
\hline 15. The minimum recommended volume of PRP is $1 \mathrm{ml}$. & 1.5 & & & 4 & 71.43 \\
\hline 16. The maximum recommended volume of PRP is $3 \mathrm{mls}$. & 1 & & & 3 & 46.43 \\
\hline 17. Leukocyte deplete PRP is the recommended formulation. & 1.5 & & & 3.5 & 50 \\
\hline 18. A single spin cycle of 20 minutes or less is recommended. & 1 & & & 4 & 64.29 \\
\hline $\begin{array}{l}\text { 19. The addition of an anticoagulant to the whole blood sample is } \\
\text { recommended prior to PRP preparation. }\end{array}$ & 1 & & & 3 & 21.42 \\
\hline $\begin{array}{l}\text { 20. PRP activation, through the addition of additives prior to its } \\
\text { administration, is not required. }\end{array}$ & 1 & & & 4 & 82.14 \\
\hline 21. Once processed, PRP should be administered within $30 \mathrm{mins}$. & 1 & & & 4 & 82.14 \\
\hline $\begin{array}{l}\text { 22. Local anaesthetic should be administered to the skin and subcutaneous } \\
\text { tissue. }\end{array}$ & 2 & & & 4 & 60.72 \\
\hline 23. Local anaesthesia should not be administered to the tendon. & 2.5 & & & 4 & 60.71 \\
\hline 24. A 19g is the recommended MAXIMUM size used to administer PRP. & 1 & & & 3 & 35.71 \\
\hline $\begin{array}{l}\text { 25. Ultrasound guidance should be utilised in all PRP injections for Lateral } \\
\text { Epicondylar Tendinopathy. }\end{array}$ & 2 & & & 4 & 67.86 \\
\hline 26. Needle fenestration is recommended over a single injection technique. & 0.5 & & & 4 & 82.14 \\
\hline $\begin{array}{l}\text { 27. Following the first administration of PRP, the patient should be reassessed } \\
\text { to discern the need for repeated administration. }\end{array}$ & 1 & & & 5 & 85.72 \\
\hline $\begin{array}{l}\text { 28. A maximum of } 3 \text { administrations is recommended for each episode of } \\
\text { Lateral Epicondylar Tendinopathy. }\end{array}$ & 2.5 & & & 3.5 & 50 \\
\hline $\begin{array}{l}\text { 29. If symptoms recur following a successful course of treatment, PRP } \\
\text { injection can be reattempted. }\end{array}$ & 1.5 & & & 4 & 75 \\
\hline $\begin{array}{l}\text { 30. Surgery is recommended for patients in whom PRP treatment is not } \\
\text { effective. }\end{array}$ & 1 & & & 4 & 71.43 \\
\hline 31. Immobilisation of the elbow following injection is not necessary. & 1 & & & 5 & 89.29 \\
\hline 32. Light loads should be avoided for the first 48 hours following injection. & 1 & & & 4 & 85.71 \\
\hline 33. Heavy loads should be avoided for 6 weeks. & 2 & & & 4 & 64.28 \\
\hline $\begin{array}{l}\text { 34. Non-Steroidal Anti-Inflammatory Drugs (NSAIDs) should be avoided for } 1 \\
\text { week prior to PRP administration. }\end{array}$ & 2 & & & 4 & 67.86 \\
\hline $\begin{array}{l}\text { 35. Non-Steroidal Anti-Inflammatory Drugs (NSAIDs) should be avoided for at } \\
\text { least } 2 \text { weeks following injection. }\end{array}$ & 2 & & & 4 & 71.43 \\
\hline $\begin{array}{l}\text { 36. Acetaminophen (Paracetamol) and weak opioid-based analgesia can be } \\
\text { offered as required following PRP administration. }\end{array}$ & 1 & & & 5 & 96.43 \\
\hline $\begin{array}{l}\text { 37. Clinical assessment is recommended to assess the outcome of PRP } \\
\text { administration. }\end{array}$ & 0.5 & & & 5 & 96.43 \\
\hline $\begin{array}{l}\text { 38. A Visual Analogue Pain Score (VAS) should be collected in addition to } \\
\text { clinical assessment. }\end{array}$ & 2 & & & 5 & 71.42 \\
\hline $\begin{array}{l}\text { 39. A validated Patient-Reported Outcome Measure (PROM)) should be } \\
\text { collected in addition to clinical assessment. }\end{array}$ & 1 & & & 4 & 82.14 \\
\hline \multirow[t]{2}{*}{ 40. Resolution of tendinosis on US or MRI can be utilised to assess outcome. } & 1.5 & & & 2 & 25 \\
\hline & & Score Key 1 & 3 & & \\
\hline
\end{tabular}


Table 1. (continued)

Statements in categories presented with interquartile range (IQR) (consensus statements highlighted in yellow), score histogram, median score and percentage agreement (a score of 4 or 5 ).

Table 2. List of statements categorised by consensus group - Consensus of: agreement, disagreement, neither agree nor disagree or consensus not reached

Statement category

\section{Consensus of agreement (median score $=4$ or 5 )}

PRP should only be considered in patients presenting with characteristic tennis elbow pain (lateral elbow pain exacerbated by wrist Patient selection extension)

PRP should only be considered in patients who are experiencing considerable intrusion into their activities of daily life

PRP treatment can be considered in patients over the age of 18 , with no upper age limit

PRP treatment can be considered in patients with manual or sedentary occupations

PRP treatment can be considered in both high demand (e.g., sports people) and low demand (e.g., office worker) patients

PRP is contraindicated in patients with known thrombocytopenia (less than 150,000 platelets per microlitre of whole blood)

A single spin cycle of 20 min or less is recommended

PRP activation, through the addition of additives prior to its administration, is not required

Once processed, PRP should be administered within $30 \mathrm{~min}$

Needle fenestration is recommended over a single injection technique

Following the first administration of PRP, the patient should be reassessed to discern the need for repeated administration

Surgery is recommended for patients in whom PRP treatment is not effective

Immobilisation of the elbow following injection is not necessary

Light loads should be avoided for the first $48 \mathrm{~h}$ following injection

Acetaminophen (paracetamol) and weak opioid-based analgesia can be offered as required following PRP administration

Clinical assessment is recommended to assess the outcome of PRP administration

A validated patient-reported outcome measure (PROM) (e.g., PRTEE, DASH, OES) should be collected in addition to clinical assessment

\section{Consensus of disagreement (median score $=1$ or 2 )}

PRP is contraindicated in patients with a coagulopathy

PRP is contraindicated in patients taking anticoagulant medication

\section{Consensus of 'neither agree nor disagree' (median score $=3$ )}

The maximum recommended platelet concentration of injected PRP is $5 \times$ baseline

The maximum recommended volume of PRP is $3 \mathrm{ml}$

The addition of an anticoagulant to the whole blood sample is recommended prior to PRP preparation

A $19 \mathrm{~g}$ is the recommended MAXIMUM size used to administer PRP

\section{Consensus not reached}

PRP should only be considered following at least 3 months of conservative therapy

PRP is contraindicated in patients with large wrist extensor tendon tears

PRP is contraindicated in patients with a dependence on non-steroidal anti-inflammatory drugs (NSAIDs)

PRP is contraindicated in patients who have received a steroid injection for treatment of their lateral epicondylar tendinopathy, within 3 months of the intended PRP treatment date

The minimum recommended platelet concentration of injected PRP is $2 \times$ baseline

The minimum recommended volume of PRP is $1 \mathrm{ml}$

Leukocyte deplete PRP is the recommended formulation

Local anaesthetic should be administered to the skin and subcutaneous tissue

Local anaesthesia should not be administered to the tendon

Ultrasound guidance should be utilised in all PRP injections for lateral epicondylar tendinopathy

A maximum of 3 administrations is recommended for each episode of lateral epicondylar tendinopathy

If symptoms recur following a successful course of treatment, PRP injection can be reattempted

Heavy loads should be avoided for 6 weeks

Non-steroidal anti-inflammatory drugs (NSAIDs) should be avoided for 1 week prior to PRP administration

Non-steroidal anti-inflammatory drugs (NSAIDs) should be avoided for at least 2 weeks following injection

A visual analogue pain score (VAS) should be collected in addition to clinical assessment

Patient selection

Patient selection

Patient selection

Patient selection

Contraindication

PRP formulation

PRP formulation

Administration technique

Administration technique

Administration strategy

Administration strategy

Post procedural care

Post procedural care

Post procedural care

Outcome assessment

Outcome assessment

Contraindications

Contraindications

PRP formulation

PRP formulation

PRP formulation

Administration technique

Patient selection

Contraindication

Contraindication

Contraindication

PRP formulation

PRP formulation

PRP formulation

Administration technique

Administration technique

Administration technique

Administration strategy

Administration strategy

Post procedural care

Post procedural care

Post procedural care

Outcome assessment

Outcome assessment 
post-procedural complications. A recent systematic review comments that the potential for unreported complications remains a major limitation of the PRP literature [26]. As a consequence of its autologous nature, there may realistically be few safety concerns, but the paucity of explicit information on adverse outcomes leaves the clinical user uninformed and simply guided by clinical, often anecdotal, experience. Though consensus was reached on PRP being contraindicated in patients with known thrombocytopaenia, consensus could not be reached on whether the presence of large wrist extensor tears, dependence on NSAIDs or a steroid injection within 3 months are contraindications, with experts providing very varied scores. Interestingly, consensus was reached on the statements regarding known coagulopathy and concurrent anticoagulation medication, with experts deeming that neither is a contraindication to PRP treatment in LET.

There have been recent calls to dramatically improve the reporting of the PRP formulation used in clinical effectiveness trials [22, 27-29]. Without this information, decisions on the plethora of PRP devices remain challenging. In this regard, it may not be surprising that limited consensus could be reached for the PRP formulation category. Consensus was gained on the use of a 20-min, or less, single spin cycle, and that the administration of additives for platelet activation was not deemed necessary. For the remaining statements, consensus of uncertainty (a median score of 3) or an inability to reach consensus was observed. Therein, the expert consensus group was unable to provide further information from their collective experience on the optimal volume, platelet concentration, addition of anticoagulant or leucocyte level. Although $71 \%$ of respondents did agree with the statement that $1 \mathrm{ml}$ of PRP was the minimum volume necessary, the overall score distribution resulted in an IQR above the a priori limit of 1 . The findings of limited consensus on PRP formulation, therefore, support the current call for improved reporting of PRP formulations, and studies undertaking formulation comparisons, in an effort to greatly enhance the knowledge base in this area.

Levels of consensus on the administration technique were equally lacking. Consensus could not be reached on the application of local anaesthetic, either to the skin/ subcutaneous tissues, or deeper into the tendon itself, whether ultrasound guidance for administration was preferable, or the optimal needle size. The needle size statement was altered between rounds two and three following the free text comments by several participants, but this did not reduce the overall dispersion of results. Consensus was reached on the requirement to deliver the PRP within 30 min of preparation, which is in accordance with a recent synthesis of available evidence [25].
Interestingly, there was consensus on the use of a fenestrated needle administration technique over a single shot approach, an aspect that currently remains controversial in the literature, with conflicting results from large systematic reviews [30, 31].

Administration strategy garnered two of four statements reaching consensus. The participants reached agreement that patients should be followed up following the first administration to discern the need for a second injection. This opposes the studies employing standardised multiple administration methodologies [32, 33], and is in accordance with the only review of multiple administration strategies conducted through retrospective cohort analysis [34]. Currently, no prospective studies have compared PRP administration strategies. Consensus was reached in favour of offering surgery for treatment failures: subgroup analysis found that surgery was favoured by surgeons, but not by the group composed of radiologists and sports physicians. Consensus was not reached concerning three injections being the maximum in one clinical episode. Further research or expert consensus measurement could be considered to assess injection numbers either side of this value. Although $75 \%$ of participants agreed that PRP could be reconsidered if symptoms were to reoccur, the responses were too widely spread to provide a clear picture, and the IQR exceeded 1.

Partial agreement was found for the post-procedural care statements. The participants did not deem immobilisation of the elbow necessary following the procedure, but $48 \mathrm{~h}$ of avoidance of light loads is recommended. However, the statement regarding avoidance of heavy loads for 6 weeks did not reach consensus and demonstrated a broad range of opinions. Further research would assist clinicians in refining this post-procedural element. Though acetaminophen (paracetamol) and weak opioid based medications were deemed appropriate post-procedural analgesics, consensus could not be reached on whether NSAIDs should be avoided 1 week prior or for 2 weeks following PRP injection. Most respondents (68\% and $71 \%$, respectively) agreed that NSAIDs should be avoided for these two periods, but there was a considerable spread of results resulting in an IQR of 2 for both statements. There are recommendations in the literature to avoid NSAIDs during this period [25], but the limited prospective evidence, once again, appears to affect the ability to derive consensus on this important post-procedural element.

Criticism from systematic reviews of PRP treatment often reference the heterogeneity or lack of validated patient reported outcome measures (PROMs) recorded as part of follow-up [11,26]. Consensus was reached in the current study on the necessity to record 
validated PROMs in addition to clinical examination. Furthermore, a strong consensus of agreement was recorded for the recommendation to conduct a clinical review of each patient undergoing PRP injection. However, consensus could not be reached on whether a visual analogue scale (VAS) was beneficial, or whether adjunct imaging (MRI or USS) could be utilised as part of the outcome assessment.

\section{Limitations}

The current study does not come without limitations. The sample size of 28 respondents represents a relatively small pool of international users of PRP injections in LET. This number would be insufficient for a simple survey but, within the iterative methodology of a Delphi study, this sample size is commensurate with published guidance on sample size to produce a representative pooling of judgments, particularly when stability between rounds can be demonstrated $[19,21]$. Efforts were made to draw a varied international sample of researchers and clinicians. However, a potential bias exists with a greater representative sample of surgeons over physicians and radiologists. Post hoc testing did not identify a disparity of agreement between these groups' statement scores, with the exception of the application of surgery as a second-line treatment. This stability of response was also demonstrated between those categorised as high-volume and lowvolume users, with no differences in statement scores.

The method of statement production, through a semi-structured questionnaire to a smaller primary round of respondents, is in keeping with previously published Delphi studies [19]. However, the authors recognise that this strategy, though well established, may well miss certain domains of interest or produce an unrepresentative stance. Nevertheless, efforts were made to represent all domains identified in previous reviews of PRP [22], and at all stages participants were able to provide free text comments if they felt domains were not represented. Furthermore, in subsequent rounds, both agreement and disagreement with these statements were assessed along with the spread of scores being utilised as the consensus criterion, as previously recommended [23]. Although percentage agreement levels are presented, and in some cases discussed above they are $>70 \%$ even though the IQR did not reach the consensus target, the authors feel that, when assessing consensus on a novel therapeutic intervention, the spread of scores, and therefore overall collective opinion, is the preferred consensus criterion, rather than a simple cut-off percentage.

\section{Conclusion}

The present study assessed the level of consensus in the applied use of platelet-rich plasma in lateral elbow tendinopathy. Although the results should not be confused with evidence of effectiveness, the findings are useful to define areas in which those with expert experience, acquired through a personal synthesis of published data and experiential practice, agree. In areas of agreement, this information can be used as clinical corroboration with reported evidence, and can guide those with more limited experience of the use of PRP in the management of LET. Obviously, what may be more relevant is where lack of consensus exists or where disagreement with manufacturers' guidance is apparent. Though consensus existed on many of the aspects of patient selection, this study has identified a striking lack of consensus on optimal PRP formulation and many aspects of the delivery techniques, reinforcing calls for improved reporting in clinical trials and a more thorough dose-dependent exploration [22, 25]. Although avoidance of PRP treatment in those with coagulopathy, drug-induced or otherwise, was not deemed necessary, the peri-procedural use of NSAIDs, steroid injections, and local anaesthetics are not held with consensus by this expert group, and require further investigation. The findings from this study support the requirement for a more structured approach to the fundamentals of PRP application and future research.

\section{Supplementary Information}

The online version contains supplementary material available at https://doi. org/10.1186/s10195-021-00608-5.

Additional file 1: Appendix S1. Medline search strategy.

Additional file 2: Appendix S2. Primary questions for round 1.

\section{Acknowledgements}

The authors would like to acknowledge the time and contribution of the experts who participated in the primary working group and Delphi survey, without whom this study would not have been possible. We would also like to thank the European Society for Surgery of the Shoulder and Elbow (SECEC), the British Association of Sports and Exercise Medicine (BASEM) and the British Society of Musculoskeletal Radiologists who distributed are invitation to participant in the study. This research was supported by the National Institute for Health Research (NIHR) Collaboration for Leadership in Applied Health Research and Care South West Peninsula. The views expressed are those of the author(s) and not necessarily those of the NHS, the NIHR or the Department of Health and Social Care. The authors would like to thank all contributors who generously volunteered their time and expertise and made this study possible.

\section{Authors' contributions}

JPE — conceived the study idea, designed the study, collected the data, performed the analysis, wrote and edited the paper. CDS—conceived the study idea, contributed data, edited the paper and approved the final manuscript. $\mathrm{NM}$ - conceived the study idea, contributed data, edited the paper and approved the final manuscript. AW—contributed data, edited the paper and approved the final manuscript. JV—conceived the study idea, edited the paper and approved the final manuscript. VG — conceived the study idea, edited the paper and approved the final manuscript. All authors read and approved the final manuscript. 


\section{Funding}

NA.

\section{Availability of data and materials}

NA.

\section{Declarations}

\section{Ethics approval and consent to participate}

This study was granted ethical approval by the University of Exeter Medical School review panel (Nov16/B/105).

\section{Consent for publication}

All authors consent to the publication of this manuscript.

\section{Competing interests}

The authors declare that they have no competing interests.

\section{Author details}

${ }^{1}$ Royal Devon and Exeter NHS Foundation Trust, Barrack Road, Exeter, UK. ${ }^{2}$ Health Services and Policy Research Group, University of Exeter, Smeal Building, St Luke's, Exeter, UK. ${ }^{3}$ Department of Musculoskeletal Disorders, University of Salerno, Salerno, Italy. ${ }^{4}$ Centre for Sports and Exercise Medicine, Barts and The London School of Medicine and Dentistry, Mile End Hospital, London, UK. Institute of Science and Technology in Medicine, Keele University School of Medicine, Thorburrow Drive, Stoke on Trent, UK. ${ }^{6}$ Wrightington Wigan and Leigh NHS Trust, Wrightington Hospital, Hill Lane, Wigan, UK. ${ }^{7}$ National Institute for Health Research (NIHR) Collaboration for Leadership in Applied Health Research and Care (CLAHRC) South West Peninsula, University of Exeter Medical School, Exeter, UK.

Received: 23 April 2021 Accepted: 31 October 2021

Published online: 25 November 2021

\section{References}

1. de Vos RJ, Windt J, Weir A (2014) Strong evidence against platelet-rich plasma injections for chronic lateral epicondylar tendinopathy: a systematic review. Br J Sports Med 48(12):952-956. https://doi.org/10.1136/ bjsports-2013-093281

2. Andia I, Latorre P, Gomez M, Burgos-Alonso N, Abate M, Maffulli N (2014) Platelet-rich plasma in the conservative treatment of painful tendinopathy: a systematic review and meta-analysis of controlled studies. Br Med Bull. https://doi.org/10.1093/bmb/ldu007

3. Nourissat G, Ornetti P, Berenbaum F, Sellam J, Richette P, Chevalier X (2015) Does platelet-rich plasma deserve a role in the treatment of tendinopathy? Joint Bone Spine 82(4):230-234. https://doi.org/10.1016/j. jbspin.2015.02.004

4. Schnabel LV, Mohammed HO, Miller BJ, McDermott WG, Jacobson MS, Santangelo KS et al (2007) Platelet rich plasma (PRP) enhances anabolic gene expression patterns in flexor digitorum superficialis tendons. J Orthop Res 25(2):230-240. https://doi.org/10.1002/jor.20278

5. McCarrel T, Fortier L (2009) Temporal growth factor release from plateletrich plasma, trehalose lyophilized platelets, and bone marrow aspirate and their effect on tendon and ligament gene expression. J Orthop Res 27(8):1033-1042. https://doi.org/10.1002/jor.20853

6. de Mos M, Koevoet W, van Schie HT, Kops N, Jahr H, Verhaar JA et al (2009) In vitro model to study chondrogenic differentiation in tendinopathy. Am J Sports Med 37(6):1214-1222. https://doi.org/10.1177/03635 46508331137

7. Anitua E, Sánchez M, Zalduendo M, De La Fuente M, Prado R, Orive G et al (2009) Fibroblastic response to treatment with different preparations rich in growth factors. Cell Prolif 42(2):162-170. https://doi.org/10.1111/j. 1365-2184.2009.00583

8. Moraes VY, Lenza M, Tamaoki MJ, Faloppa F, Belloti JC (2014) Platelet-rich therapies for musculoskeletal soft tissue injuries. Cochrane Libr. https:// doi.org/10.1002/14651858.CD010071.pub2

9. Ahmad Z, Brooks R, Kang SN, Weaver H, Nunney I, Tytherleigh-Strong $G$ et al (2013) The effect of platelet-rich plasma on clinical outcomes in lateral epicondylitis. Arthroscopy 29(11):1851-1862. https://doi.org/10. 1016/j.arthro.2013.07.272

10. Krogh TP, Bartels EM, Ellingsen T, Stengaard-Pedersen K, Buchbinder R, Fredberg U et al (2013) Comparative effectiveness of injection therapies in lateral epicondylitis: a systematic review and network meta-analysis of randomized controlled trials. Am J Sports Med 41(6):1435-1446. https:// doi.org/10.1177/0363546512458237

11. Long L, Briscoe S, Cooper C, Hyde C, Crathorne L (2015) What is the clinical effectiveness and cost-effectiveness of conservative interventions for tendinopathy? An overview of systematic reviews of clinical effectiveness and systematic review of economic evaluations. Health Technol Assess. https://doi.org/10.3310/hta19080

12. Kaux JF, Bouvard M, Lecut C, Oury C, Gothot A, Sanchez M et al (2015) Reflections about the optimisation of the treatment of tendinopathies with PRP. Muscles Ligaments Tendons J 5(1):1-4

13. Linstone HA, Turoff M (1975) The Delphi method: techniques and applications. Addison-Wesley Reading, MA

14. Delbecq AL, Van de Ven AH, Gustafson DH (1975) Group techniques for program planning: a guide to nominal group and Delphi processes. Scott Foresman, Glenview

15. Powell C (2003) The Delphi technique: myths and realities. J Adv Nurs 41(4):376-382

16. Murphy M, Black N, Lamping D, McKee C, Sanderson C, Askham J et al (1998) Consensus development methods, and their use in clinical guideline development: a review. Health Technol Assess 2(3):88

17. O'Neill S, Watson PJ, Barry S (2016) A Delphi study of risk factors for Achilles tendinopathy-opinions of world tendon experts. Int J Sports Phys Ther 11(5):684

18. Eubank BH, Mohtadi NG, Lafave MR, Wiley JP, Bois AJ, Boorman RS et al (2016) Using the modified Delphi method to establish clinical consensus for the diagnosis and treatment of patients with rotator cuff pathology. BMC Med Res Methodol 16(1):56. https://doi.org/10.1186/ s12874-016-0165-8

19. Hsu C-C, Sandford BA (2007) The Delphi technique: making sense of consensus. Pract Assess Res Eval 12(10):1-8

20. Ludwig B (1997) Predicting the future: have you considered using the Delphi methodology. J Ext 35(5):1-4

21. Hasson F, Keeney S, McKenna H (2000) Research guidelines for the Delphi survey technique. J Adv Nurs 32(4):1008-1015

22. Murray IR, Geeslin AG, Goudie EB, Petrigliano FA, LaPrade RF (2017) Minimum information for studies evaluating biologics in orthopaedics (MIBO): platelet-rich plasma and mesenchymal stem cells. JBJS 99(10):809-819. https://doi.org/10.2106/JBJS.16.00793

23. Heiko A (2012) Consensus measurement in Delphi studies: review and implications for future quality assurance. Technol Forecast Soc Chang 79(8):1525-1536. https://doi.org/10.1016/j.techfore.2012.04.013

24. Kalaian SA, Kasim RM (2012) Terminating sequential Delphi survey data collection. Pract Assess Res Eval 17(1):5

25. LaPrade RF, Geeslin AG, Murray IR, MusahI V, Zlotnicki JP, Petrigliano F et al (2016) Biologic treatments for sports injuries II think tank — current concepts, future research, and barriers to advancement, Part 1: Biologics overview, ligament injury, tendinopathy. Am J Sports Med 44(12):32703283. https://doi.org/10.1177/0363546516634674

26. Miller LE, Parrish WR, Roides B, Bhattacharyya S (2017) Efficacy of plateletrich plasma injections for symptomatic tendinopathy: systematic review and meta-analysis of randomised injection-controlled trials. BMJ Open Sport Exerc Med. https://doi.org/10.1136/bmjsem-2017-000237

27. DeLong JM, Russell RP, Mazzocca AD (2012) Platelet-rich plasma: the PAW classification system. Arthrosc J Arthrosc Relat Surg 28(7):998-1009. https://doi.org/10.1016/j.arthro.2012.04.148

28. Magalon J, Chateau A, Bertrand B, Louis M, Silvestre A, Giraudo L et al (2016) DEPA classification: a proposal for standardising PRP use and a retrospective application of available devices. BMJ Open Sport Exerc Med 2(1):e000060. https://doi.org/10.1136/bmjsem-2015-000060

29. Ehrenfest DMD, Andia I, Zumstein MA, Zhang C-Q, Pinto NR, Bielecki T (2014) Classification of platelet concentrates (platelet-rich plasma-PRP, platelet-rich fibrin-PRF) for topical and infiltrative use in orthopaedic and sports medicine: current consensus, clinical implications and perspectives. MLTJ Muscles Ligaments Tendons J 4(1):3-9

30. Fitzpatrick J, Bulsara M, Zheng MH (2017) The effectiveness of plateletrich plasma in the treatment of tendinopathy: a meta-analysis of 
randomized controlled clinical trials. Am J Sports Med 45(1):226-233. https://doi.org/10.1177/0363546516643716

31. Dong W, Goost H, Lin X-B, Burger C, Paul C, Wang Z-L et al (2016) Injection therapies for lateral epicondylalgia: a systematic review and Bayesian network meta-analysis. Br J Sports Med 50(15):900-908. https://doi.org/ 10.1136/bjsports-2014-094387

32. Creaney L, Wallace A, Curtis M, Connell D (2011) Growth factor-based therapies provide additional benefit beyond physical therapy in resistant elbow tendinopathy: a prospective, double-blind, randomised trial of autologous blood injections versus platelet-rich plasma injections. Br J Sports Med 45(12):966-971. https://doi.org/10.1136/bjsm.2010.082503

33. Stenhouse G, Sookur P, Watson M (2013) Do blood growth factors offer additional benefit in refractory lateral epicondylitis? A prospective, randomized pilot trial of dry needling as a stand-alone procedure versus dry needling and autologous conditioned plasma. Skeletal Radiol 42(11):1515-1520. https://doi.org/10.1007/s00256-013-1691-7

34. Glanzmann MC, Audigé $L$ (2015) Platelet-rich plasma for chronic lateral epicondylitis: Is one injection sufficient? Arch Orthop Trauma Surg 135(12):1637-1645. https://doi.org/10.1007/s00402-015-2322-7

\section{Publisher's Note}

Springer Nature remains neutral with regard to jurisdictional claims in published maps and institutional affiliations.

\section{Submit your manuscript to a SpringerOpen ${ }^{\circ}$ journal and benefit from:}

- Convenient online submission

- Rigorous peer review

- Open access: articles freely available online

- High visibility within the field

- Retaining the copyright to your article

Submit your next manuscript at $\boldsymbol{\nabla}$ springeropen.com 